\title{
CAPTURA Y RECAPTURA DE MOSCA DEL ESTABLO EN GANADO Y RASTROJOS DE PIÑA1
}

Yannery Gómez-Bonilla², Arturo Solórzano Arroyo², Oscar Bravo Bonilla²

\section{RESUMEN}

Captura y recaptura de mosca del establo en ganado y rastrojos de piña. El objetivo de este estudio fue conocer el movimiento de la mosca del establo del ganado hacia el rastrojo de piña y viceversa. Se realizó captura, marcado, liberación y recaptura de $S$. calcitrans en rastrojos de piña y ganaderías en la localidad de Pital, San Carlos, Alajuela, Costa Rica. El marcado de los insectos se realizó mediante el uso de polvos indelebles color fluorescente. Se realizó un experimento en mayo del año 2013 donde se capturaron y liberaron en rastrojo de piña 763 moscas del establo. En este estudio se recapturan moscas a los (3, 7 y 14) días a $(100,700$ y 500) metros de distancia del rastrojo de piña. El segundo experimento se realizó en setiembre del mismo año, donde se liberaron en rastrojo de piña 577 moscas con polvo fluorescente naranja, y en el ganado 176 moscas de color azul. Se recapturaron cuatro moscas marcadas de azul a los tres días a los (200, 400 y 900) metros y dos color naranja a los 5 y 21 días a (300 y 1000) metros entre el rastrojo de piña y ganado. Los dos experimentos cumplen con la distribución de Poisson. Utilizando el método de Peterson-Lincon, se estimó el desplazamiento por metro, donde los Límites de Confianza al 95 \%, indican que el estudio de mayo en rastrojo de piña, se puede encontrar de 3 a 43 moscas y en el de setiembre en rastrojo de piña 2 a 197 moscas y en ganado de 3 a 24 moscas. La captura y recaptura demostró el movimiento de la mosca del establo desde el rastrojo de piña (donde se reproduce) hasta el ganado (donde se alimenta), y se trasladó entre $100 \mathrm{~m}$ a 2,3 km.

Palabras clave: Stomoxys calcitrans, plaga de ganado, método de captura y recaptura, mosca marcada.

1 Este trabajo formó parte de un proyecto de investigación financiado por el INTA y la Fundación para el Fomento y Promoción de la Investigación y Transferencia de Tecnología Agropecuaria de Costa Rica (FITTACORI).

2 Instituto Nacional de Innovación y Transferencia en Tecnología Agropecuaria. INTA. Costa Rica. ygomez@inta.go.cr, asolorzano@inta.go.cr, obravo@inta.go.cr. Sede del laboratorio de Servicios de Fitoprotección del INTA. Sabana Sur, San José. 


\section{INTRODUCCIÓN}

La mosca del establo (Stomoxys calcitrans) es una plaga reconocida mundialmente por su impacto en la ganadería (Foil y Hogsette 1994; Kunz et al. 1991, Taylor y Berkebile 2006), en países como Estados Unidos, genera pérdidas anuales por el orden de más de dos mil millones de dólares (Taylor et al. 2012). En la ganadería costarricense también se considera una plaga importante, los primeros reportes del insecto se realizaron durante el año 1987 (Herrero 1991), y posteriormente los brotes se han incrementado en la última década (Solórzano et al. 2013).

Esta mosca es un parásito externo, hematófago, ampliamente distribuido y que afecta el ganado de carne y leche. En altas poblaciones causa efectos adversos, tanto por la pérdida de sangre, como por las molestias e irritación que provocan (Cruz-Vázquez et al. 2000). Las picaduras de la mosca del establo son dolorosas, afectando directamente los animales, provocando que se agrupen, y por ende aumenta el estrés por calor (Foil y Hogsette 1994) disminuye la ganancia de peso y la producción de leche (Taylor et al. 2012), afectando también, la vitalidad del animal y horas de pastoreo (Harwood y James 1979, Campbell et al. 2001).

En estudios de dispersión de mosca del establo utilizando adultos marcados, se ha logrado evidenciar la dirección y recorrido (Gersabeck y Merrit 1985, Scholl 1986, Taylor et al. 2010). Se ha observado que tanto machos como hembras se dispersaron en la misma distancia y dirección, un $50 \%$ se encontró hasta $1,6 \mathrm{~km}$ del punto de liberación, pero únicamente un $5 \%$ a más de 5,1 $\mathrm{km}$ de distancia (Taylor et al. 2010). En un estudio similar, se demostró que un $50 \%$ de la población liberada en la Isla de Mackinag-Michichan-USA se recapturó a 0,45 km de distancia y el $90 \%$ a $1,65 \mathrm{~km}$, desde el punto de liberación, sin lograr recapturar ningún ejemplar en tierra firme a 11,4 km de distancia (Gersabeck y Merritt 1985). En otra investigación el $80 \%$ de las moscas liberadas fueron recuperadas entre dos lotes separados a $0,8 \mathrm{~km}$ de distancia (Scholl 1986). A pesar de que hay reportes de largas distancias recorridas de hasta $225 \mathrm{~km}$ en las costas del noroeste de Florida (Hogssette et al. 1985), se considera que la mosca del establo no se mueve mucho en la búsqueda de sangre o sitio de oviposición, ya que los vuelos registrados de larga distancia de esta plaga han sido atribuidos por fenómenos ambientales como asistido por el viento por ejemplo (Showler y Obsbrink 2015).

La mosca del establo es reconocida por su capacidad de desarrollarse en materiales vegetativos en descomposición sobre todo aquellos contaminados con estiércol y orina (Talley et al. 2009). La mezcla del estiércol maduro de las lecherías con pasto, son reservorios muy adecuados para el desarrollo de la misma (Meyer y Petersen 1983, Gilles et al. 2005, Taylor y Berkebille 2011). Desde hace más de una década, se ha determinado que los principales focos de esta plaga que afectan la ganadería en los Estados Unidos, provienen de las áreas de pastoreo y sitios de alimentación con cultivos forrajeros durante la época de invierno (Broce 1988, Taylor et al. 2010). No obstante, en Costa Rica se ha comprobado que, al triturar las plantas de piña, se produce algún tipo de atrayente para la mosca del establo, y en pocas horas, la misma llega a reproducirse y ovipositar en el rastrojo (Solórzano et al. 2013, Solórzano et al. 2015, Vargas y Solórzano 2016.

El impacto que ha tenido esta sobrepoblación de mosca del establo sobre el ganado ha sido muy contraproducente, dicha situación es particular, ya que mundialmente la plaga se desarrolla en explotaciones pecuarias, no como el caso que se describe, en el cual la plaga se reproduce principalmente en un cultivo agrícola e impacta las ganaderías cercanas al área de cultivo. El objetivo del presente estudio fue identificar la capacidad de dispersión de la mosca del establo del ganado hacia el rastrojo de piña y viceversa. 


\section{MATERIALES Y MÉTODOS}

\section{Área de estudio}

El estudio se realizó en la empresa Agrícola Industrial La Lydia, ubicada en la localidad de Pital, distrito de San Carlos, Costa Rica. Se realizaron dos pruebas: una en mayo y otra en setiembre del 2013. Para estos experimentos se empleó una parcela de $13 \times 100 \mathrm{~m}\left(1300 \mathrm{~m}^{2}\right)$ de una plantación de piña con dos años de edad (alrededor de 8500 plantas). Se trituraron plantas de piña (rastrojo fresco), utilizando una máquina trituradora (Seppi Midiforst M DT 150m HP a 5000 rpm), bajo el sistema de derriba en verde (plantas de piña después de cosechar los frutos y deshija, sin aplicación de herbicidas secantes). En el caso de la ganadería, se localizó el ganado en pastoreo, que estuviera más cercano al lugar de liberación. En ambos estudios los sitios de estudio fueron georeferenciados con un equipo GPS Garmin 60S.

\section{Captura y liberación de moscas del establo}

Para la captura de adultos de mosca del establo, se emplearon trampas de tela de color azul y negro modelo NZI (Figura 1) derivadas de trampas para la captura de mosca del sueño Tse Tsé (Glosina spp.), evaluadas en rastrojos de piña para la captura de mosca del establo (Solórzano et al. 2015). Las trampas tenían forma triangular con un panel de color azul de 1,6 $\mathrm{m}^{2}$ y una pantalla negra de 0,9 $\mathrm{m}^{2}$ (Mihok 2002, Gilles et al. 2007). Se colocaron cinco trampas sobre el lote de piña recién triturado. Se utilizaron polvos fluorescentes indelebles resistentes al agua, de los colores azul, naranja y fucsia o rojo púrpura en frascos de vidrio transparentes con tapa, de un litro de capacidad, se colocaron aproximadamente $20 \mathrm{~g}$ de polvo fluorescente indeleble (DyeGlow ${ }^{\circledR}$ ) para marcar las moscas. El frasco con el polvo se introdujo dentro de la trampa NZI hasta donde las moscas se encontraban atrapadas vivas ubicadas dentro de la trampa, las mismas se recogían con el frasco y se tapaba para evitar el escape. Las moscas dentro del frasco se impregnaban del colorante (Figura 2), posteriormente se liberaron. El polvo en el frasco se iba rellenando conforme se acababa.

La captura de la mosca del establo se hizo cada media hora, desde las 8,00 a.m. hasta las 5,00 p.m. El primer experimento (mayo), las moscas se capturaron en el cultivo triturado de piña y se marcaron con colorante fucsia. En el segundo experimento (setiembre), las moscas capturadas en el cultivo triturado con piña se marcaron con el colorante naranja y las capturadas cerca del ganado en pastoreo, se marcaron con el colorante azul (Figura 3).

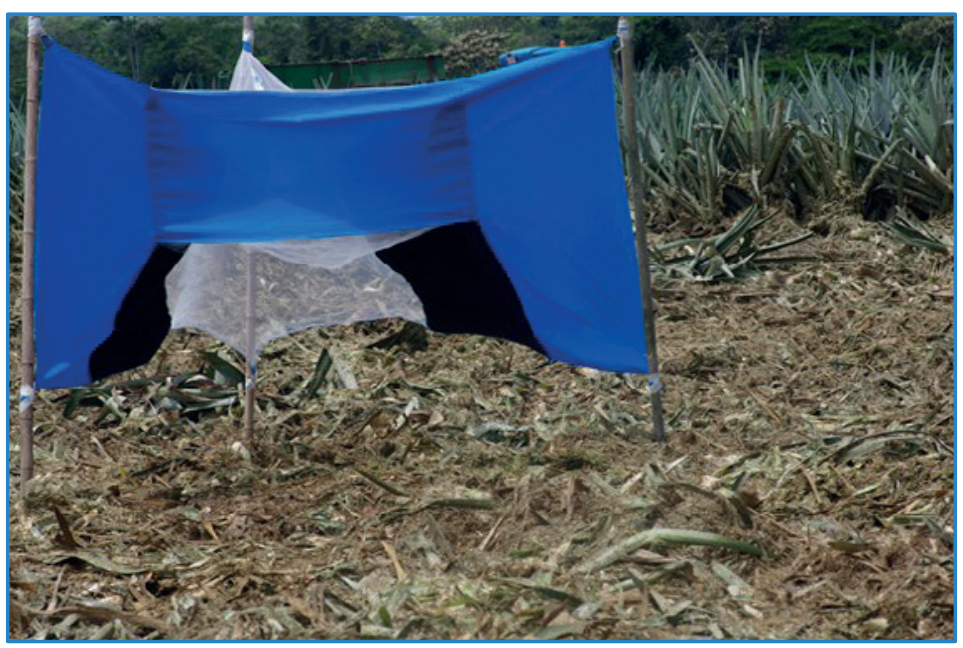

Figura 1. Trampa modelo NZI para captura y marcación de moscas del establo sobre plantas de piña trituradas. Pital de San Carlos, Alajuela, Costa Rica. 2013. 

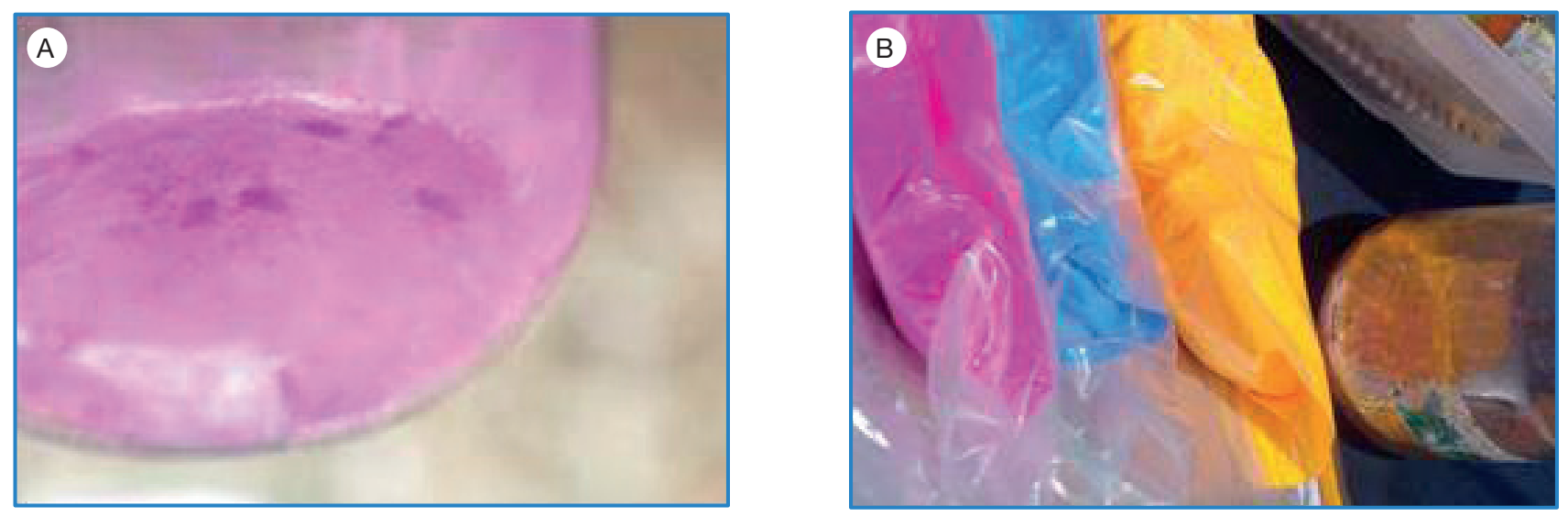

Figura 2. A. Frasco de vidrio con colorante fluorescente y mosca del establo marcada. B. Polvos fluorescentes utilizados en el experimento para marcado de moscas del establo. Pital de San Carlos, Alajuela, Costa Rica. 2013.

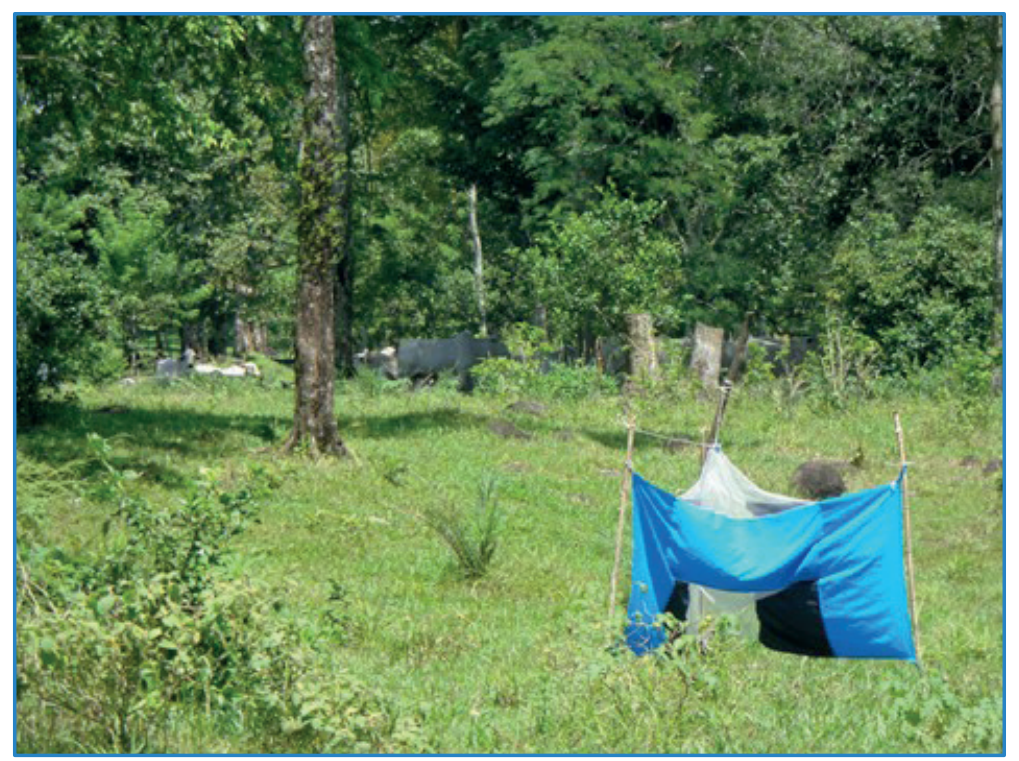

Figura 3. Trampas NZI en ganado pastoreando para la captura de mosca del establo viva. Pital de San Carlos, Alajuela, Costa Rica. 2013. 


\section{Recaptura de moscas del establo}

Para la recaptura, desde el primer día se colocaron 60 trampas con bolsas blancas (Figura 4) pintadas con pegamento Zalpicol (diluido 1:1 con gasolina) las mismas se ubicaron sobre dos palos de madera a $20 \mathrm{~cm}$ del suelo. La distancia empleada entre trampas varió según la topografía del terreno, pero osciló entre $100 \mathrm{~m}$ para las primeras tres trampas desde el sitio de liberación y $300 \mathrm{~m}$ para las siguientes. La dirección tomada fue en los cuatro puntos cardinales, hasta $5 \mathrm{~km}$ de distancia y próximas a las ganaderías circundantes. Los puntos de colocación de las trampas fueron georreferenciados, dándose el respectivo seguimiento una vez ubicadas. Después de liberadas las moscas, todos los días durante 5 días, se dio seguimiento a las trampas blancas para la recaptura, se realizó un conteo de las moscas atrapadas en las bolsas, se anotó la cantidad total de moscas del establo y de moscas marcadas con polvo fluorescente. Las bolsas blancas con pegamento fueron cambiadas todos los días. Terminado este periodo de cinco días, se dejaron las bolsas blancas con pegamento por una semana. Cada ocho días las bolsas blancas fueron cambiadas por nuevas bolsas, esto se realizó durante tres semanas.

Para la recolecta de las bolsas blancas se colocó un plástico transparente sobre ellas, para ser llevadas al laboratorio. En el laboratorio en una habitación oscura se revisaron con lámpara de luz ultravioleta.

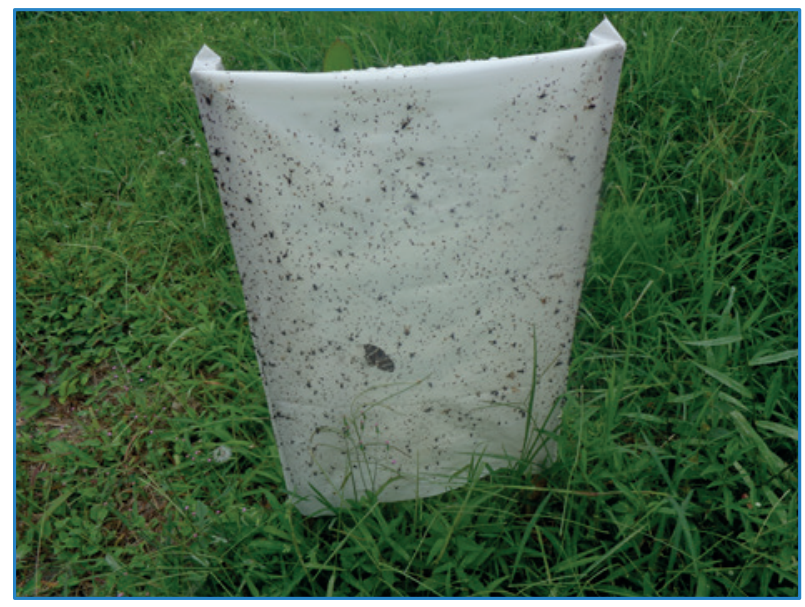

Figura 4. Trampa con bolsa blanca pintada con pegamento, sostenida por dos palos a $20 \mathrm{~cm}$ del suelo. Pital de San Carlos, Alajuela, Costa Rica. 2013.

\section{Análisis de datos}

Para determinar el desplazamiento de la población por metro, en el análisis de datos se utilizó el Método de Lincoln-Peterson (Badii et al. 2012), que es el que mejor se ajustaba a las características de este experimento, con este método se asume que es una población cerrada y que todos los miembros de la población tienen la misma probabilidad de ser marcados y recapturados y que los insectos marcados se distribuyen al azar en la población. Se calculó el Intervalo de Confianza (IC) para los límites inferior y superior al $95 \%$ el cual se obtiene con la siguiente fórmula: $\dot{N}=[(\mathrm{M}+1)(\mathrm{C}+1) /(\mathrm{R}+1)]-1$. Con base en esta información se construyó el Cuadro 1, donde se estimó el desplazamiento a un $95 \%$ de confiabilidad de las moscas por metro para cada una de las poblaciones.

$\dot{N}=C M / R$.

$\mathrm{M}=$ número de individuos marcados en la primera muestra.

$\mathrm{C}=$ número de individuos capturados en el segundo muestreo.

$\mathrm{R}=$ número de individuos con marcaje en el segundo muestreo.

$\dot{N}=$ estimación del tamaño de la población al tiempo de poner la marca. 


\section{RESULTADOS Y DISCUSION}

La captura y recaptura demostró el movimiento de la mosca del establo desde el rastrojo de piña (donde se reproduce) hasta el ganado (donde se alimenta de sangre) y viceversa. Se trasladó entre $100 \mathrm{~m}$ a 2,3 km, en un radio menor a $5 \mathrm{~km}$. En el experimento realizado en mayo 2013 se capturaron y liberaron 763 moscas provenientes de los rastrojos de piña, donde la mosca llega a ovipositar. En la Figura 5, se muestra en color rojo el punto central donde fueron liberadas las moscas, el color magenta en el mapa representa las tres moscas recapturadas. En dirección al ganado en pastoreo (noreste) a 100 metros del punto de liberación, se encontró una mosca marcada el primer día (Figura 6). En dirección sur-oeste, se recaptura una mosca a $700 \mathrm{~m}$ ocho días después, y por último otra mosca a $500 \mathrm{~m}$ a los catorce días, dirección a la lechería, que se encontraba a $2290 \mathrm{~m}$ del punto de liberación, donde la mosca llega a alimentarse. Esta población tiene una distribución de Poisson y según el Método de Lincoln-Peterson, se determinó con un Intervalo de Confianza (IC) al $95 \%$ (Cuadro 1), que se pueden encontrar de ( 3 a 43 moscas) por metro (Cuadro 1) en el sitio de estudio, según (Balestrieri et al. 2016; Badii et al. 2012; Brownie et al. 1993), el método da una estimación bastante precisa del tamaño de población. Durante las tres semanas de evaluación, se presentaron días lluviosos $(1,4,7,9,11$ y 12$)$ con precipitaciones de entre 5 y $75 \mathrm{~mm}$ y temperaturas que variaron entre 24 a $33{ }^{\circ} \mathrm{C}$ (Figura 7 ), este tipo de condiciones con días alternos entre lluvia y sol, son muy favorables porque permite que se descomponga la planta de piña y atrae a la mosca para que llegue a reproducirse (Solórzano et al. 2015) y permite la dispersión de la mosca.

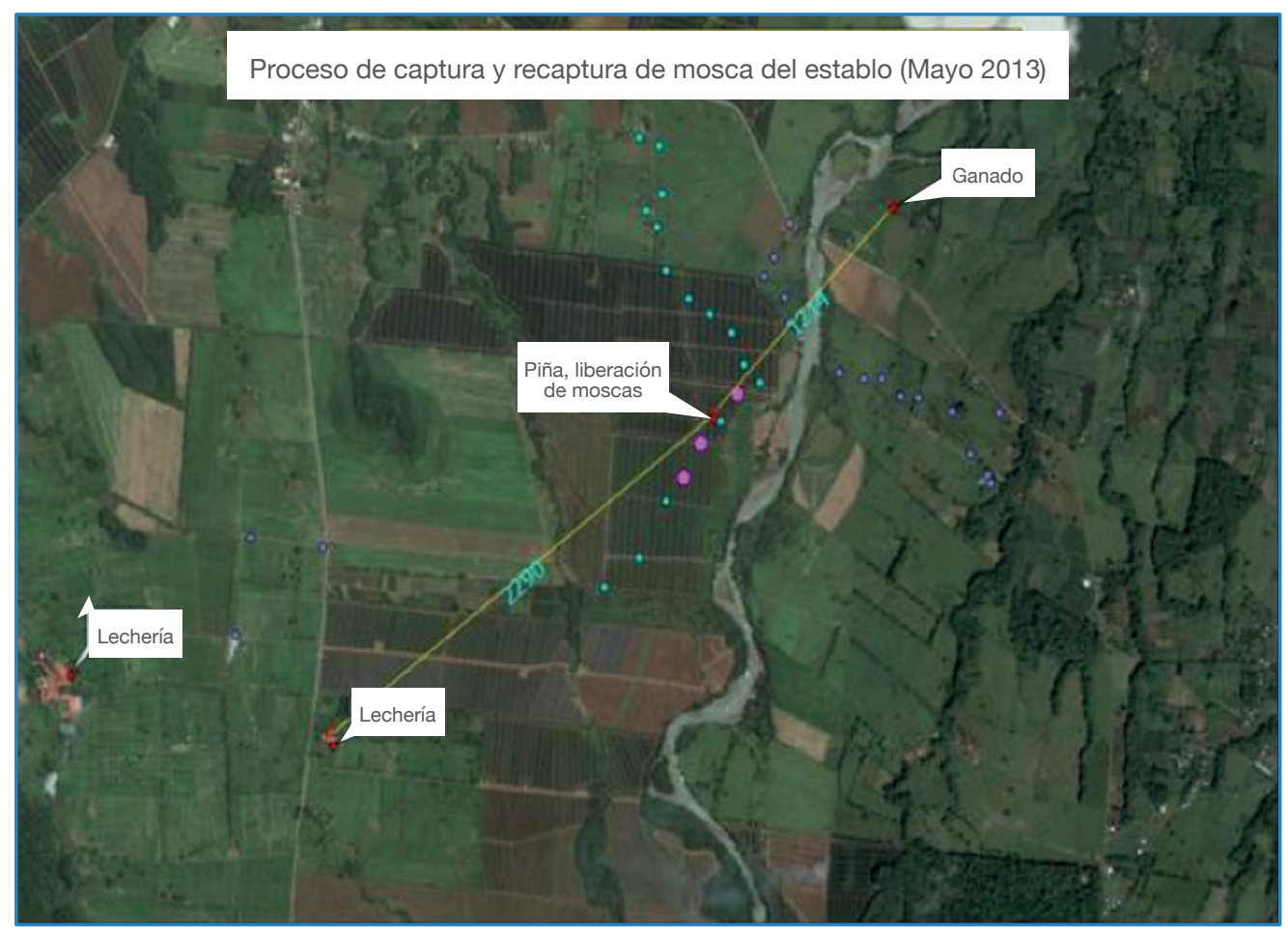

Figura 5. Cultivo de piña, mapa de liberación y recaptura de mosca del establo, marcadas con polvo fluorescente Pital de San Carlos, Alajuela, Costa Rica. Mayo, 2013. 


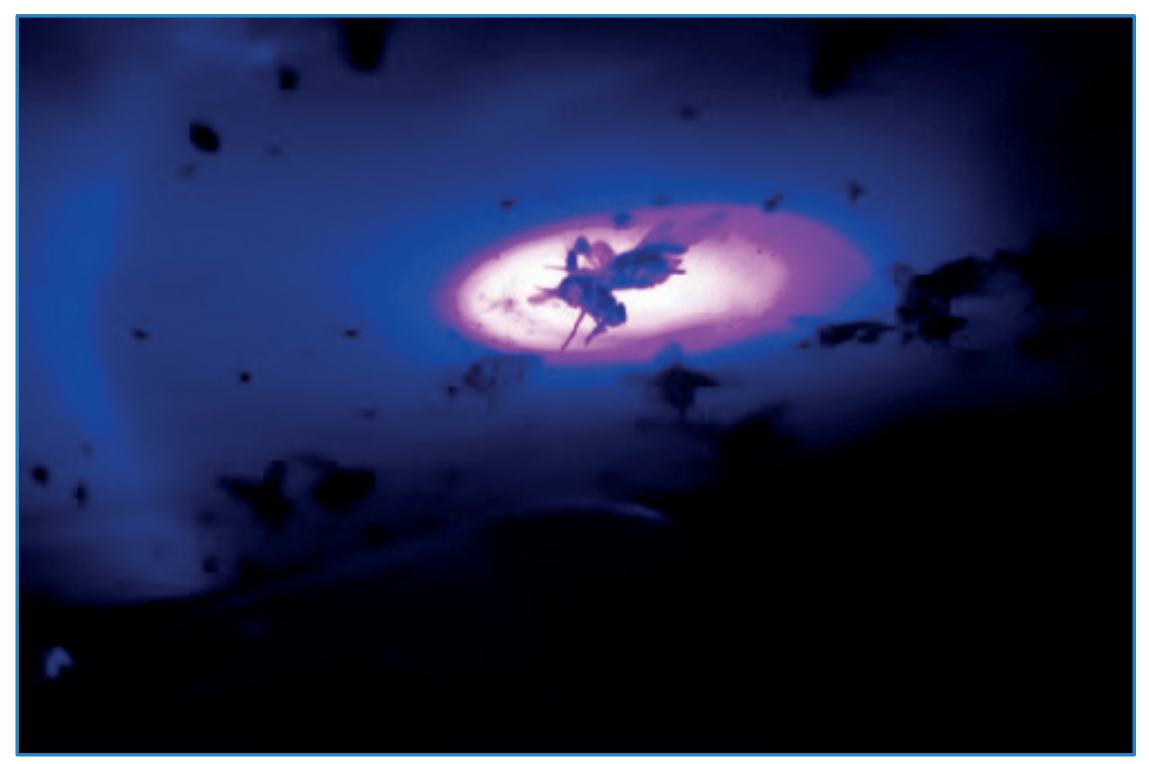

Figura 6. Mosca del establo marcada con polvo fluorescente color fucsia, alumbrada con lámpara de luz ultravioleta de alta intensidad. Pital de San Carlos. 2013.

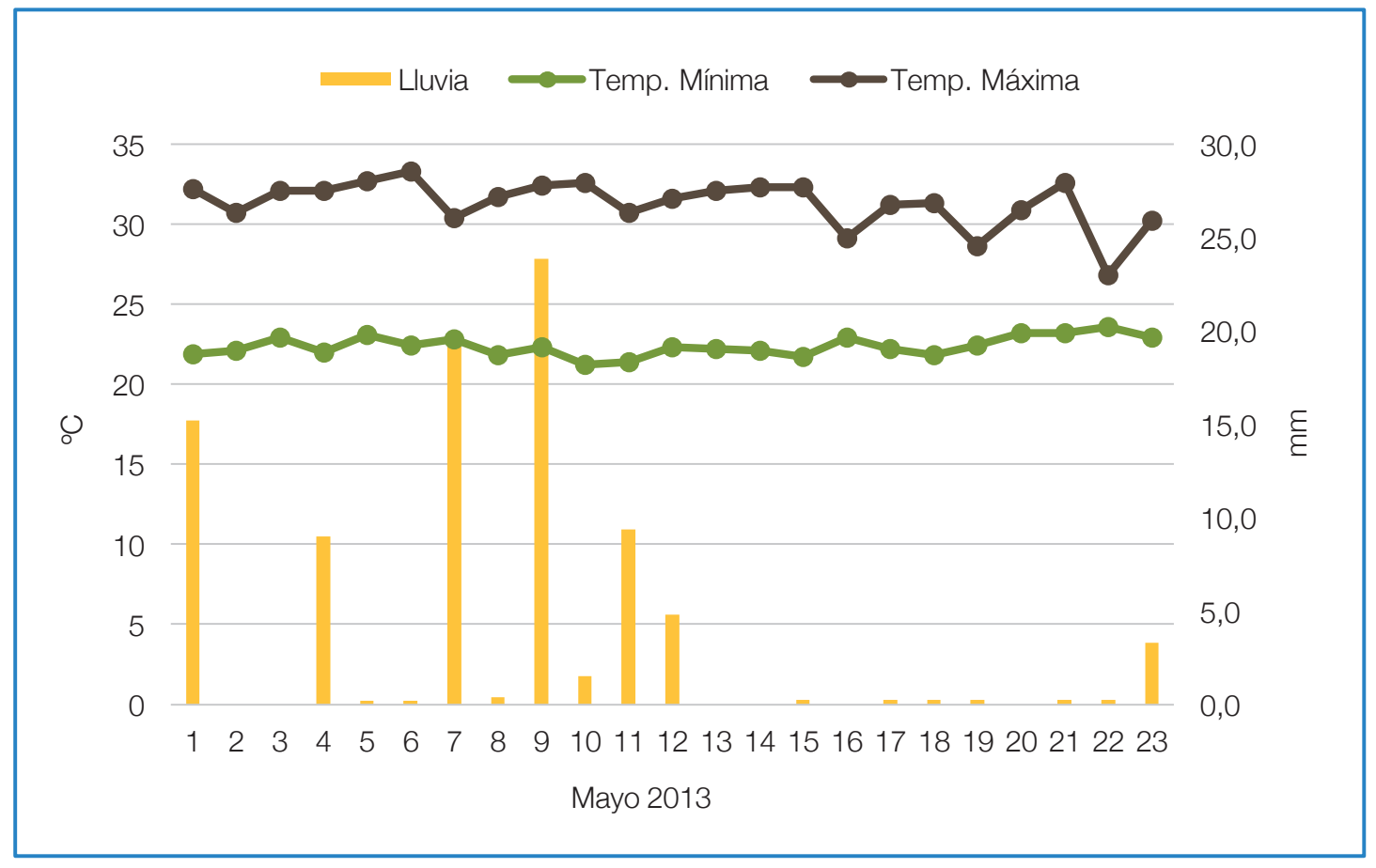

Figura 7. Datos de clima diarios en Pital de San Carlos, Alajuela, Costa Rica. Mayo del 2013. 
En el segundo estudio (Figura 8), 577 moscas fueron liberadas del rastrojo de piña marcadas de color naranja, y 176 moscas en total marcadas de color azul, de este total se capturó y liberó en la ganadería en pastoreo 120 moscas y de la lechería 56 moscas. De las moscas marcadas color naranja se recapturaron dos una aproximadamente a los cinco días a $500 \mathrm{~m}$, cerca del rastrojo de piña (dirección suroeste) y la otra aproximadamente a $1000 \mathrm{~m}$ de la ganadería en pastoreo (dirección noreste) a los 21 días después de liberada, distancia entre puntos $2155 \mathrm{~m}$. Se recapturaron todas las moscas marcadas en azul, a los tres días de liberadas. Del rastrojo de piña (dirección suroeste) tres moscas color azul a 200, 400 y $900 \mathrm{~m}$, posiblemente proveniente de la lechería, distancia de $1587 \mathrm{~m}$ entre ambos puntos y la otra mosca color azul (noreste) a $200 \mathrm{~m}$ posiblemente proveniente del ganado de pastoreo. Estas poblaciones también tienen distribución de
Poisson y según el Método de Lincoln-Peterson, se determinó con un Intervalo de Confianza (IC) al $95 \%$ (Cuadro 1), que, en el caso del rastrojo de piña, se puede encontrar de (2 a 197 moscas) por metro y en el caso de la población del ganado (3 a 24 moscas) por metro.

Durante las cuatro semanas del estudio se presentó nueve días sin lluvia y algunos días con altas precipitaciones diarias superior a los $20 \mathrm{~mm}$ (Figura 9) condiciones apropiadas para la oviposición y vuelo de la mosca del establo en rastrojos de piña (Solórzano et al. 2013) así como para permitir el vuelo para la ingesta de sangre. Las temperaturas oscilaron entre 19 y $34^{\circ} \mathrm{C}$ las cuales fueron apropiadas para permitir el desarrollo de adultos de mosca del establo, completar su ciclo de vida y seguir ovipositando (Salem et al. 2012, Vargas y Solórzano 2016, Gilles 2007).

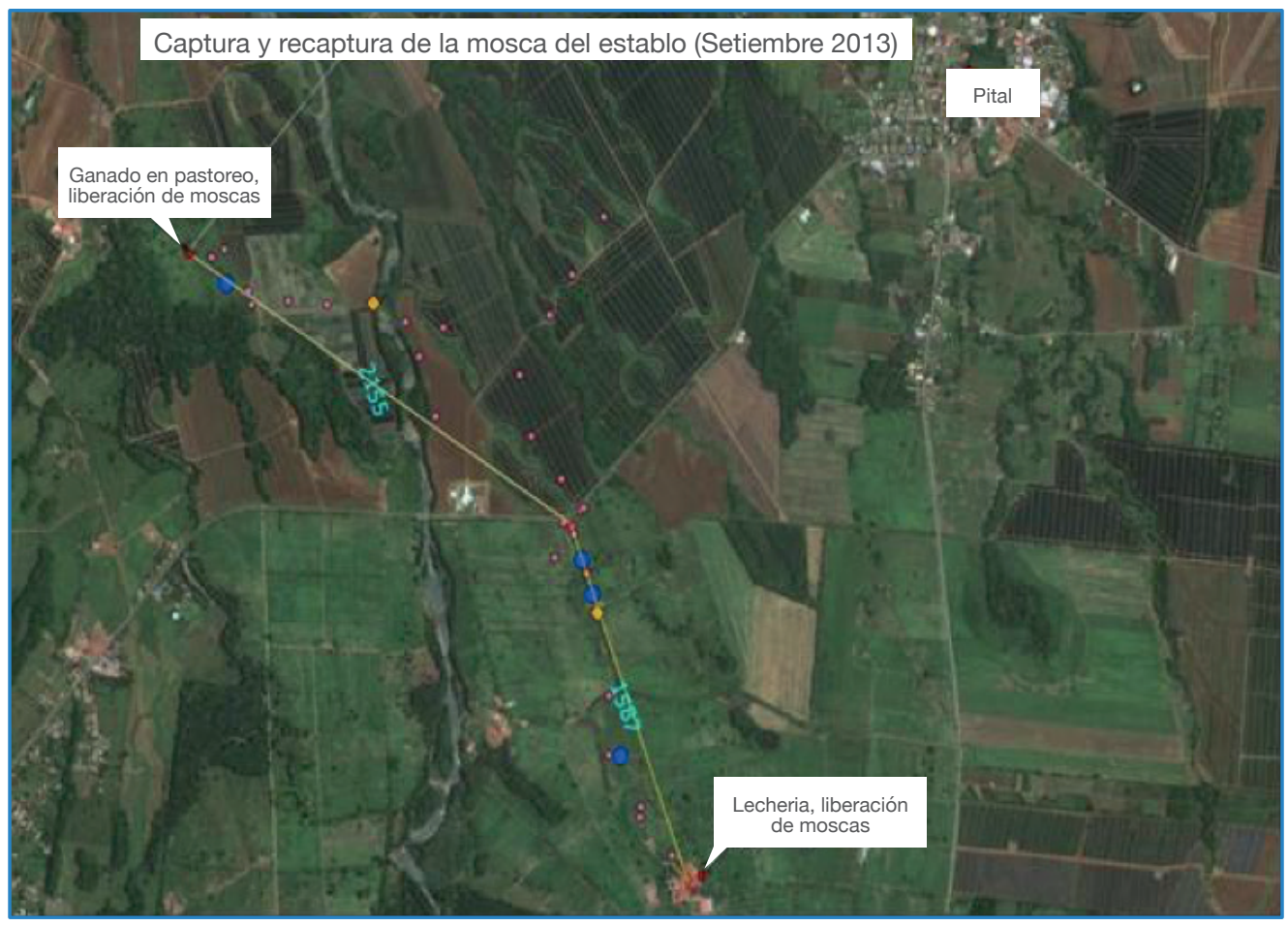

Figura 8. Cultivo de piña, mapa de liberación y recaptura de mosca del establo marcadas con polvo fluorescente. Pital de San Carlos, Alajuela, Costa Rica. Setiembre, 2013. 


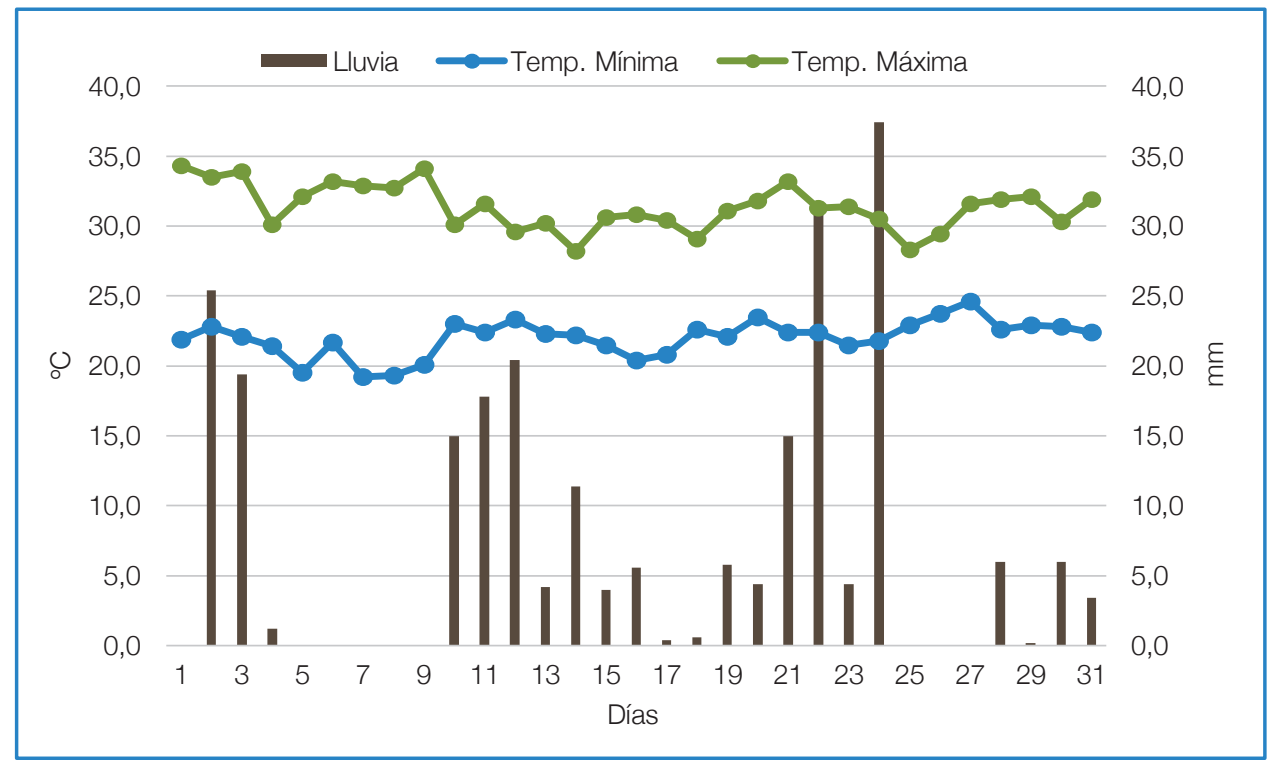

Figura 9. Datos de clima entre setiembre-octubre del 2013. Pital de San Carlos, Alajuela, Costa Rica. Datos proporcionados por PINDECO.

Cuadro 1: Estimación de la densidad de mosca del establo por metro en los tres sitios de estudio y sus Intervalos de Confianza (IC). Pital de San Carlos, Alajuela, Costa Rica. 2013.

\begin{tabular}{l|c|c|c|} 
& $\begin{array}{c}\text { Tamaño } \\
\text { de } \\
\text { pobla- } \\
\text { ción/ } \\
\text { metro }\end{array}$ & \multicolumn{2}{|c}{$\begin{array}{c}\text { Intervalos de } \\
\text { confianza (IC)/ m }\end{array}$} \\
\cline { 3 - 4 } & $\begin{array}{r}95 \% \text { LI. } \\
\text { (Límite inferior) }\end{array}$ & $\begin{array}{r}95 \% \text { LS. } \\
\text { (Límite superior) }\end{array}$ \\
\hline $\begin{array}{l}\text { Población } \\
\text { de rastrojo } \\
\text { piña-mayo }\end{array}$ & 23 & 3 & 43 \\
\hline $\begin{array}{l}\text { Población de } \\
\text { rastrojo } \\
\text { piña-setiembre }\end{array}$ & 99 & 2 & 197 \\
\hline $\begin{array}{l}\text { Población } \\
\text { ganado- }\end{array}$ & 14 & 3 & \\
setiembre & & & \\
\hline
\end{tabular}

En los estudios realizados del ciclo de vida de la mosca del establo, se ha reportado que la mosca adulta en el laboratorio ha alcanzado hasta veintiocho días viva (Vargas y Solórzano 2016), veinticuatro días (Salem et al. 2012) o hasta cuarenta días a $25{ }^{\circ} \mathrm{C}$, con agua y miel (Gilles et al. 2005). Sin embargo, Taylor et al. (2014) señalan que, en condiciones de campo en verano, Nebraska, Estados Unidos, la sobrevivencia no es mayor a un par de semanas, debido a las condiciones extremas de temperatura y humedad relativa. La mosca del establo en condiciones de clima templado, el tiempo medio de supervivencia de los adultos fue de 6,3 días, que van desde 4,3 hasta 11,1 días (Skovgard y Nachman 2012).
En este estudio bajo condiciones de trópico, la duración de vida del adulto hasta la recapturada fue, en el primer estudio (mayo) de catorce días y en el segundo (setiembre) de veintiún días (las moscas fueron capturadas vivas y no se conoce el tiempo que tenían de haber salido de pupa), muy aproximado con el ciclo producido en laboratorio (Vargas y Solórzano 2016) considerando que las hembras empiezan a ovipositar entre los ocho y nueve días y los adultos capturados en rastrojos de piña recién triturados son en su mayoría hembras grávidas (Solórzano et al. 2015).

Los polvos fluorescentes y el método de captura y recaptura son alternativas que contribuyen a generar el conocimiento sobre el comportamiento del díptero, lo que permite darle seguimiento a este tipo de especies (Grigoreva 1992, Charlwood y Sama 1997, Taylor et al. 2010). En el trabajo realizado por (Charlwood y Sama 1997) recapturaron siete de 312 moscas marcadas, al día siguiente de la liberación. En el estudio de (Gersabeck y Merritt 1985) mencionan que el $90 \%$ de las moscas se recuperaron a menos de $0,8 \mathrm{~km}$ de los sitios de liberación, e indican que otros factores además de la dirección del viento influyeron en su dispersión. Los mismos autores citan a (Bailey et al. 1973) quién especuló que una vez que las moscas del establo encontraron una fuente de sangre, tienden a alimentarse y descansar en el área varios días. En un estudio sobre la dinámica de la mosca del establo (Taylor et al. 2010), encontraron que el $50 \%$ de la población se dispersó a 1,6 km de distancia y solo el $5 \%$ a $5,1 \mathrm{~km}$. En este estudio como se 
mencionó anteriormente la distancia recorrida fue de 100 a 2,3 km.

La mosca del establo es una plaga que se reproduce en el material en descomposición del rastrojo del cultivo piña y no perjudica al cultivo, por lo tanto, el productor piñero considera que no debe invertir en su control. Lo contrario pasa con los ganaderos y sus semovientes, el problema se da principalmente en el ganado de carne y leche, pero afecta también a caballos, perros y otros. La decisión de implementar medidas de prevención depende de la dificultad de emprender una acción alterna, o de la ineficacia de las medidas extemporáneas de combate (Coto y Saunders 2004), y más aún, el incurrir en un gasto para el combate de una plaga que no afecta al cultivo de piña, permite pensar al productor que no hay razón para buscar medidas de control.

\section{AGRADECIMIENTOS}

A la finca la Lydia por haber facilitado lotes de piña para realizar las investigaciones. A PINDECO, por facilitar los datos climatológicos. A FITTACORI por la ayuda económica. A las ganaderías cercanas que nos permitieron tomar los datos. Al Dr. David Taylor USDA-ARS por la donación de los polvos para marcar insectos

\section{LITERATURA CITADA}

Badii, MH; Guillen, A; Landeros, J; Cerna, E; Ochoa, Y; Valenzuela, J. 2012. Muestreo por Métodos de Captura-Recaptura. International Journal of Good Conscience. 7(1):97-131.

Balestrieri, A; Zenato, M; Fontana, E; Vezza, P; Remonti, L; Caronni, FE; Saino, N; Prigioni, C. 2016. An indirect method for assessing the abundance of introduced pest Myocastor coypus (Rodentia) in agricultural landscapes (en línea). Journal of Zoology. 298(1):37-45. Consultado 16 nov. 2017. Disponible en doi:10.1111/jzo.12284.

Broce, AB. 1988. An improved Alsynite trap for stable flies, Stomoxys calcitrans (Diptera: Muscidae). J. Med. Entomol. 25(5):406-409.

Brownie, C; Hines, JE; Nicholls, K; Pollock, KH; Hestbeck, JB. 1993. Statistical inference for capture-recapture experiments. BIOMETRICS. 49(4):1173-1187

Campbell, J; Skoda, S; Berkebile, D; Boxler, R; Thomas, D; Adams, D; Davis, R. 2001. Effects of stable flies (Diptera: Muscidae) on weight gain of grazing yearling cattle. J. Econ. Entomol. 94(3):780-783.

Charlwood, JD; Sama, C. 1997. The age structure, biting cycle and dispersal of Stomoxys niger Macquart (Diptera: Muscidae) from Ifakara, Tanzania. Afr. Entomol. 4(2):274-277.
Cisneros, VF. 1995. Control de plagas agrícolas. Lima, Perú, Centro Internacional de la Papa. 475 p.

Coto, D; Saunders, JL. 2004. Insectos plagas de cultivos perennes con énfasis en frutales en Amércica Central. Turrialba, Costa Rica, CATIE. $399 \mathrm{p}$.

Cruz-Vázquez, C; Martínez-Rangel, S; VitelaMendoza, I; Ramos-Parra, M; Quintero-Martínez, MT; García-Vázquez, Z. 2000. Variación anual de la infestación por Stomoxys calcitrans (L.) (Diptera: Musicidae) en tres establos lecheros de Aguascalientes. Tec. Pecu. Méx. 38(2):135-142.

Foil, LD; Hogsette, JA. 1994. Biology and control of tabanids, stable flies and horn flies. Rev. Sci. Tech. Off. Int. Epiz. 13(4):1125-1158.

Gersabeck, EF; Merritt, RW. 1985. Dispersal of adult Stomoxys calcitrans (L.) (Diptera: Muscidae) from known immature developmental areas. J. Econ. Entomol. 78(3):617-621.

Gilles, J; David, J; Duvallet, G. 2005. Effects of temperature on the rate of increase of Stomoxys calcitrans and Stomoxys niger niger (Diptera: Muscidae) from La Reunion Island. J. Med. Entomol. 42(6):959-965. 
Gilles, J; David, J; Duvallet, G; De la Rocqueand, S;Tillard, E. 2007. Efficiency of traps for Stomoxys calcitrans and Stomoxys niger niger on Reunion Island. Med Vet. Entomol. 21(1):65-69.

Grigoreva, LA. 1992. Absolute numbers of Stomoxys calcitrans in dairy farm buildings. Parazitologiya. 26:430-435.

Harwood, RF; James, MT. 1979. Entomology and human animal health. N.Y., USA, McMillan Publishing. 548 p.

Herrero, MV; Montes-Pico, L; Hernández, R. 1991. Relative abundance of Stomoxys calcitrans (L.) (Diptera: Muscidae) in six localities on the southern Pacific coast of Costa Rica. Rev. Biol. Trop. 39(2):309-310.

Hogsette, J; Ruff, J. 1985. Stable fly (Diptera: Muscidae) migration in northwest Florida. Environ. Entomol.14(2)170-175.

Kunz, SE; Murrell, KD; Lambert, G; James, LF; Terrill, CE. 1991. Estimated losses of livestock to pests. In: Pimentel, D. (Ed), CRC Handbook of Pest Management in Agriculture, vol. 1. CRC Press, Boca Raton, FL, USA. p. 69-98.

Meyer, JA; Peterson, JJ. 1983. Characterization and seasonal distribution of breeding sites of stable flies (Diptera: Muscidae) on eastern Nebraska feedlots and dairies. J. Econ. Entomol. 76(1):103-108.

Mihok, S. 2002. The development of a multipurpose trap (the NZI) for tse-tse and other biting flies. Bull. Entomol. Res. 92(5): 385-403.

Salem, A; Franc, M; Jaquiet, M; Bouhsiraand, E; Lienard, E. 2012. Parasite. Feedinf and breeding aspects of Stomoxys calcitrans (Diptera: Muscidae) under laboratory conditions. Parasite. 19(4):309-317.

Scholl, P. 1986. Field populations of Stomoxys calcitrans (L) in Eastern Nebraska. Southwest Entomol. 9:155-160.

Showler, A; Osbrink, W. 2015. Stable Fly, Stomoxys calcitrans (L.), Dispersal and Governing Factors. International Journal of Insect Science. 2015(7):19-25.

Skovgard, H; Nachman, G. 2012. Population dynamics of stable flies Stomoxys calcitrans (Diptera: Muscidae) at an organic dairy farm in Denmark based on mark-recapture with destructive sub-sampling. Environ. Entomol. 41(1):20-29.
Solórzano, J; Treviño, J; Hidalgo, E; GómezBonilla, Y; Blanco, H; Apuy, M; González, L; Meneses, D. 2013. Recomendaciones para el manejo de la mosca del establo Stomoxys calcitrans en el cultivo de piña. Memorias Taller manejo de rastrojos del cultivo de piña y plagas que afectan la competitividad. San Carlos, Costa Rica, PITTA PIÑA. 32 p.

Solórzano, JA.; Gilles, J; Bravo, O; Vargas, C; Gómez-Bonilla, Y; Bingham, GV; Taylor, D. 2015. Biology and Trapping of Stable Flies (Diptera: Muscidae) Developing in Pineapple Residues (Ananas comosus) in Costa Rica (en línea). J. Insect Sci. 15(1):145-150. Consultado 11 de may. 2017. Disponible en: doi: 10.1093/jisesa/iev127.

Talley, JL.; Broce, AB; Zurek, L. 2009. Characterization of stable fly (Diptera: Muscidae) larval development habitat at round hay bale feeding sites. J. Med. Entomol. 46(6):1310-1319.

Taylor, DB; Friesen, K; Zhu, JJ. 2014. Stable Fly Control in Cattle Winter Feeding Sites with Novaluron. Arthropod Manag. Tests. 39(1):30-40.

Taylor, D; Moon, R; Marck, D. 2012. Economic impact of stable fly (Diptera: Muscidae) on beef cattle production. J. Med. Entom. 49(1):198-209.

Taylor, D; Berkebile, DR. 2011. Phenology of stable fly (Diptera: Muscidae) larvae in Round Bale Hay feeding sites in Eastern Nebraska (en línea). Environ. Entomol. 40(2):84-193. Consultado 02 de may. 2017. Disponible en: doi: 10.1603/EN10245.

Taylor, DB; Moon, RD; Campbell, JB; Berkebile, DR; Scholl, PJ, Broce, AB; Hogsette, JA. 2010. Dispersal of stable flies (Diptera: Muscidae) from larval development sites in a Nebraska landscape (en línea). Environ. Entomol. 39(4):1101-1110. Consultado 02 de may. 2017. Disponible en: doi: 10.1603/EN10057.

Taylor, D; Berkebile, D. 2006. Comparative efficiency of six stable fly (Diptera: Muscidae) traps. J. Econ. Entomol. 99(4):1415-1419.

Vargas, C; Solórzano, A. 2016. Biología y cría de mosca del establo Stomoxys calcitrans $L$. Rev. Alcances Tecnológicos-INTA, Costa Rica. 11(1):5-19. 